\title{
State of monitoring and evaluation in Anglophone Africa: Centre for Learning on Evaluation and Results in Anglophone Africa's reflections
}

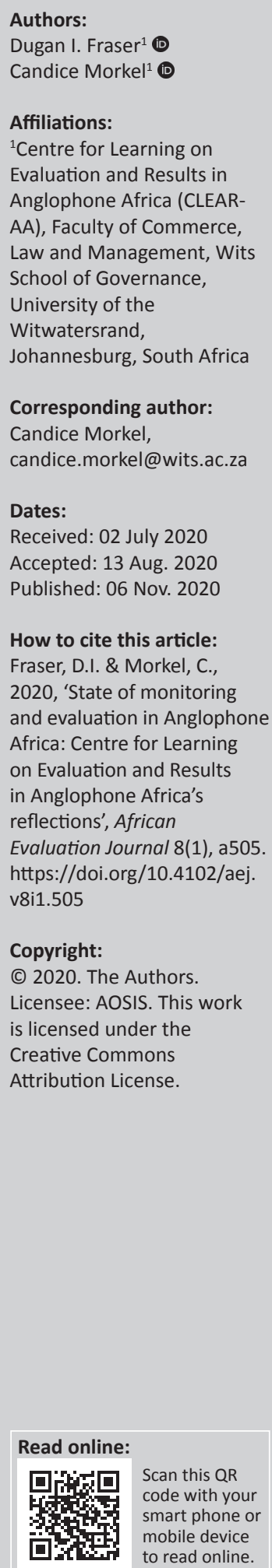

This article is an overview of what the Centre for Learning on Evaluation and Results in Anglophone Africa (CLEAR-AA) is currently learning in its work implementing monitoring and evaluation (M\&E) capacity strengthening programmes in our partner countries. This article is based on the reflections drawn from the authors' experiences and the work of CLEARAA in strengthening M\&E systems across the continent. It serves as a contribution to larger ongoing strategic conversations about how to promote evidence-informed decision-making for better development outcomes. The article begins with a discussion on systems broadly and M\&E systems in particular, with a specific focus on some of the historical roots of the current ways in which M\&E is defined and implemented in African systems of governance. We continue to discuss the various elements that come into play in establishing and institutionalising M\&E systems, in particular the 'M\&E Market' and the demand for evidence, where we also challenge the notion of the unidirectional demand and supply chain of evaluation. The institutional architecture within which M\&E systems operate is next discussed, and how the formal (and informal) laws, policies, boundaries and rules continue to provide some degree of leverage in support of these systems. The article finally addresses two key elements of developing and sustaining M\&E systems: the role of leadership and developing an evaluative culture. The authors explain why these elements, which often receive less attention than the technical elements in building and strengthening M\&E systems, carry such weight in sustaining national evaluation systems.

Keywords: Evaluation systems; Evaluation capacity development; Leadership; Evaluation culture; Evaluation management systems; Evaluation supply and demand.

\section{Introduction}

The Centre for Learning on Evaluation and Results in Anglophone Africa (CLEAR-AA) is one of the six regional centres in the CLEAR Initiative working globally to strengthen capacity to undertake monitoring and evaluation (M\&E) and to use evidence to improve programmes and support policymakers and implementers in making better decisions. It has been operational for nearly 10 years, focusing on African countries in which English is an official language, and has developed a rich body of experience and an extensive network of relationships that give it valuable insights into the M\&E capacity development endeavour.

At the time of writing this article, we are being faced with one of the most significant global events in the history of the current generation of people inhabiting the earth. The Coronavirus Disease 2019 (COVID-19) pandemic has set for the world's leaders the gargantuan task of ensuring that good evidence leads their decision-making around securing the safety of and saving the lives of millions of people. More than ever before, governments around the world need systems of evidence that will enable them to make the right decisions rapidly. The potentially catastrophic effects that may come about as a result of the absence of good evidence (or the use of bad evidence) highlight, now more than ever, the critical demand for stronger, more effective M\&E systems, particularly at the level of the state.

Although the literature on a system approach to building national M\&E systems is fairly nascent, there is an emerging body of knowledge that we can begin to draw from. One of these is a book, published by Routledge and available in July 2020, ${ }^{1}$ entitled Using Evidence in Policy and Practice:

1.Available from https://www.routledge.com/Using-Evidence-in-Policy-and-Practice-Open-Access-Lessons-from-Africa/Goldman-Pabari/p/ book/9780367440077 and other booksellers. 
Lessons from Africa edited by Ian Goldman and Mine Pabari. Based on a case-study approach of five countries and the Economic Community of West African States (ECOWAS) (West African) region and drawing from a range of sectors and types of evidence used, the book provides some insight into how African governments can use evidence to the benefit of their citizens by improving decision-making, policymaking and programme implementation. Works such as these are important in ensuring that we build a robust body of knowledge to guide our work in partnering with governments (and non-state actors) across the African continent in strengthening national M\&E systems.

As a further contribution to this growing body of knowledge, this article is an overview of what CLEAR-AA is currently learning in its work implementing M\&E capacity strengthening programmes in our partner countries. This article is based on the reflections drawn from the authors' experiences and the work of CLEAR-AA in strengthening M\&E systems in the continent. It serves as a contribution to larger ongoing strategic conversations about how to promote evidence-informed decision-making for better development outcomes. The article begins with a discussion on systems broadly and M\&E systems in particular, with a specific focus on some of the historical roots of the current ways in which $M \& E$ is defined and implemented in African systems of governance. We continue to discuss the various elements that come into play in establishing and institutionalising M\&E systems, in particular, the 'M\&E Market' and the demand for evidence, where we also challenge the notion of the unidirectional demand and supply chain of evaluation. The institutional architecture within which M\&E systems operate and the ways the formal (and informal) laws, policies, boundaries and rules continue to provide some degree of leverage in support of these systems are discussed next. The article finally addresses two key elements of developing and sustaining M\&E systems: the role of leadership and developing an evaluative culture. The authors explain why these elements, which often receive less attention than the technical elements in building and strengthening M\&E systems, carry such weight in sustaining national evaluation systems (NESs).

This article is limited by the anecdotal nature of the observations presented here and concludes with a number of lessons that have been learnt as part of our work on the continent. These include the importance of collaborative partnerships in working at scale, of embedding M\&E in broader institutional systems of decision-making, the critical role that leadership plays in providing momentum to (and sustaining) efforts at institutionalising M\&E, as well as the growing of African-centred approaches to build and strengthen local and regional evaluation systems.

\section{The integration of monitoring and evaluation into management systems}

A system-based approach to M\&E strengthening is essential, as the utility of M\&E almost always relies on the extent to which systems for collecting, synthesising, analysing, reporting and using evidence are integrated into systems for policy-making, planning, learning and adaptation. Monitoring and evaluation are often treated as adjuncts to policy, programme and project implementation, but to add real value, they need to be integrated from the outset into the management planning and decision-making processes and components of institutional systems. In generic terms, a system usually refers to an interdependent group of items forming a unified whole (Arnold \& Wade 2015). At an institutional level, specifically one in which systems of evidence production and use operate, we recognise that this includes the enabling environment, the human and nonhuman, technical and financial elements that make up the system, as well as rules (formal and informal) about how decisions are made and actions are taken. Although our work focuses primarily on these elements, we are also mindful of their interconnectedness to broader local and global sociopolitico-economic systems, and the potential influence of the various parts on each other.

There has been significant growth in the efforts to establish M\&E systems and functions in governments, particularly in the Global South. Countries, such as South Africa, Tanzania and Uganda (amongst others), have built M\&E systems to assess various strategies and national development plans (CLEAR-AA 2013). One of the reasons for this is the pressure on governments to implement their Sustainable Development Goals (SDGs), and report on their performance in the periodic Voluntary National Reviews (VNRs) presented by Heads of State to the High-Level Political Forum at the annual United Nations General Assembly (United Nations c. 2020). One of the effects of the growth in M\&E systems is a shift from accounting for budget expenditure to a focus on the achievement of development results, which is a welcome development. Monitoring and evaluation now needs to be located in the broader discourse around sustainable development and the achievement of systemwide development outcomes.

However, the maturity of both M\&E is uneven across the African continent, where systems of routine monitoring of performance exist, even if they are often poorly curated and managed. The bifurcation and decoupling of monitoring from evaluation are worsened by the widespread split between policy, planning and M\&E structures and functions. In many administrations, ministries of finance (or treasuries) remain primarily responsible for fiscal planning and budgeting, which they do with little or no engagement with entities responsible for the generation and utilisation of evidence. This concurs with Masuku (2015), highlighting that government departments work in silos and other entities are often not included. Government sectors take their cue from decisions about programme delivery made by national fiscal and sector planning processes, and evaluation findings rarely find their way into the budget decision-making system. One key observation has been that there are prevailing weaknesses in national systems for the collection and management of 
administrative data, and in some cases, even basic recordkeeping on the delivery of public goods is poorly managed. These systems are often treated as entirely separate from initiatives to build NESs, which contribute to weaknesses in integrated governance and accountability, and ultimately the broader concerns of evidence-informed policy-making.

For developing countries, implementing results-based management is costly and requires time to plan and develop (Pazvakavambwa \& Steyn 2014), and there is an ongoing demand to strengthen results-based management practices in the public sector. Although public sector strategic planning, goal-setting and prioritisation are implemented, these processes often miss key elements that would make the underlying programme logic visible. These include accounts of the mechanisms by which change is intended to happen, as well as the risks and assumptions that underlie programmes' theories of change. Many of the fundamental principles of M\&E, such as developing theories of change for all programmes or interventions, are still not part of planning and budgeting vocabularies and thus need to be routinised and promoted.

Policies informed by a 'New Public Management' orientation and visions for development that prioritise economic development have shaped the way M\&E operates in contemporary African society. In the case of South Africa, for example, the pursuit of a capable, efficient and effective state has created a distinctly hierarchical form of governance, which propels a compliance and oversight-driven approach to M\&E. In fact, research on the state and use of M\&E systems in the South African national and provincial government suggested 'a rather narrow use of M\&E for internal monitoring and control' (Umlaw \& Chitepo 2015:13). These audit-oriented processes create perverse incentives, where senior government executives chase the certainty of quick wins and 'low hanging fruits' over complex outcomes.

In addition to strengthening public governance and administration systems, there needs to be more room for nonstate actors in government M\&E practices. Representatives of civil society, academic institutions and other producers of evidence are rarely part of institutional planning, performance monitoring, evaluation and improvement processes, and there are few mechanisms to include their evidence in policymaking, planning and budgeting. In research around the coproduction of public services by Fledderus, Brandsen and Honing (2014), the relationship between government and civil society is based on the general posture or orientation of the state towards co-production. The inclusion of outside voices requires an embracing of a collaborative approach by the state and the nurturing of closer, more cordial relationships between government and civil society, which can help to strengthen collaboration (Tsai 1999:70). Addressing M\&E at a system level, therefore, requires that we consider broad M\&E ecosystems and become more inclusive in our approaches towards institutionalisation.

\section{The monitoring and evaluation 'market': Supply and demand}

In the M\&E context, supply refers to those who produce evidence, whilst demand refers to those who use M\&E products or services. In government-led evaluation systems, policymakers or programme leaders could be thought of as representing the demand side and evaluation consultants representing the supply side. However, one could also think of demand as the donor agencies that commission evaluations on programmes they run in partner countries, or even supply, when they co-produce evaluations with sub-contracted consultants for use in government policy and decisionmaking. Therefore, we can also make a distinction between supply and demand as it is expressed within a government M\&E system versus supply and demand within the broader ecosystem.

Twende Mbele's (2018) three-country study on supply and demand revealed that the nature of demand for and supply of M\&E goods and services varies widely depending on the country context. For example, demand is driven largely by donors in Benin, whereas government largely drives demand in South Africa (Twende Mbele 2019a). A number of factors interact together to shape the supply and demand chain rather than a linear cause-and-effect process (Blaser Mapitsa \& Khumalo 2018:4). Weaknesses in supply are, therefore, not necessarily because of weaknesses in the skills and capabilities of the supply side. The Twende Mbele study (Blaser Mapitsa \& Khumalo 2018:4) as well as analyses conducted by CLEARAA, such as the Compass, ${ }^{2}$ suggested that the tendency to use external, international evaluators was a policy preference, rather than being caused by shortages on the local supply side (Blaser Mapitsa \& Khumalo 2018:4).

However, the problem is often articulated as one of supply - that there are not enough good evaluators to meet the demand. The diagnostic report on supply and demand (Phillips 2018), part of the Twende Mbele study, highlights that supply may be seen as inadequate in relation to demand as in some cases submitted evaluation reports must be reworked by government officials. On the other hand, the study inferred that the supply of evaluators may actually be adequate to meet demand and that the real problem lies with the demand side. In some cases, evaluators choose not to work with government because of the bureaucratic burden, onerous procurement procedures and overbearing management of evaluations by government clients. The poor quality of evaluation specifications has also been raised, both in the Twende study and in the evaluation of the South African NES undertaken in 2017 (Department of Planning, Monitoring and Evaluation [DPME] 2017:107). One way that Twende Mbele (2019) recommends remedying the misalignment between supply and demand is to

2.In 2017 and 2018, CLEAR-AA conducted a cross-country comparison study of M\&E systems in Anglophone Africa and produced 'The Compass'. The first Compass was produced in 2017 and constituted of 11 countries. These countries were Benin Botswana, Cote d'Ivoire, Ghana, Kenya, Niger, South Africa, Tanzania, Uganda, Botswana, Cote d'Ivoire, Ghana, Kenya, Niger, South Africa, Tanzania, Uganda,
Zambia and Zimbabwe. The second Compass was produced in 2018, and focuses on six Anglophone Countries; Uganda, Ghana, Kenya, South Africa, Rwanda and Zambia. 
encourage researchers within academic institutions to conduct evaluations, although it is not convincing whether this would be an adequate response to the more systemic challenges.

Despite these factors, most literature on African evaluations highlights weaknesses in supply (Merkle 2016:4; Podems, Goldman \& Jacob 2014). Studies on evidence supply and demand conducted in 2005 and 2014 both indicated the popular belief in the weak individual capacities for evaluation and the poor quality of evaluation training (Abrahams 2015:4; Podems et al. 2014:76). Capacity building seems to hinge on the ability to produce more and better evidence, but the issue of integrating that evidence into evaluation systems is not widely considered. The issue of evidence utilisation is frequently raised as a concern in our work in strengthening NESs and the pre-occupation with just strengthening the capacities of individual evaluators needs reframing.

Our own work in building NESs has led us to move away from thinking about linear supply chains. We are increasingly adopting a system perspective in our country strategies, where we recognise the need for diverse, tailored packages of interventions that include, but are not limited to, building individual capacities. Although the demand continues to be presented as a need for training, we seek to combine this with technical assistance, policy support, institutional system development, the creation of enabling environments and the building of individual capacities. In working together with country partners, there is greater appreciation of the need to view capacity strengthening in a more holistic and integrated manner, which builds in-country sustainability in the area of $\mathrm{M} \& \mathrm{E}$, and can (in a sense) be thought of as capacity building through the process of engagement. We have also developed a real appreciation of the limits individual organisations face in strengthening country M\&E systems. Specialised organisations that focus on, for example, certain kinds of evaluation or training can have a catalytic impact on strengthening parts of the system, but no single intervention, on its own, can constitute the whole solution. The work of CLEAR-AA (and indeed the work of the CLEAR network as a whole) is, therefore, built on a highly collaborative approach, and our partnership with a multitude of organisations (both state and non-state, local and international) is an intentional aspect of our practice.

The recognition that partnerships are essential to achieve success in strengthening the M\&E evidence production and use value chain is gaining traction in the sector, and we have seen a global shift towards such collaborations (such as the Global Evaluation Initiative, in which the CLEAR network is a key element). Such efforts recognise that collaborative partnerships are essential to achieve success at scale (particularly in the light of the urgency towards the achievement of the SDGs as well as other pressing global challenges such as the COVID-19 and climate crises).

\section{Enabling environments: Laws, policies and practices}

In any context, government-driven M\&E systems reflect country traditions and dynamics, are shaped and constrained by the state and social architecture in which they function, are driven by local political and ideological dynamics and are enabled and limited by the prevailing human and financial capacities. The Centre for Learning on Evaluation and Results in Anglophone Africa (2019a:6) states that, in many ministries, there is institutional architecture which is a foundation for the production and use of evaluation within government.

Few Anglophone African countries have formal policy or legislation guiding the practice of M\&E. Uganda, South Africa, Zambia, Botswana, Zimbabwe and Nigeria are the Anglophone countries with approved M\&E policies, whilst Kenya and Ghana have draft policies awaiting formal adoption. Whilst some evaluations are undertaken in certain English-speaking African countries, these are mostly commissioned by donors or multilateral agencies (rather than the state), with public agencies playing a limited role in their commissioning or utilisation. Goldman et al. (2018:7) provided evidence of donors, highlighting the roles that donors play; in South Africa, donors are scarcer and do not form a part of the M\&E system; in Benin, evaluations are funded by donors; and in Uganda, donors are integrated within the system, whilst financing and involvement in the oversight mechanisms are done by donors. Even in countries where the legislation or policy is clear, implementation is often patchy, and those administrations need to rethink elements of their approach in order to achieve the widespread evidence-informed decisionmaking sought through their policies.

Monitoring practices and processes in the countries where CLEAR-AA works are characterised by a common feature: they seldom operate across internal organisational boundaries, making it hard for decision-makers and policymakers to get a view of a whole sector or system, and encourage a silo perspective that limits the ability to plan and think systemically as required to address complex challenges. In most instances, the data systems used for performance reporting are paper-based and where they are digital, they use very basic software without the necessary governance policies to ensure interoperability or to protect privacy. In Zambia, challenges with performance monitoring are intensified by the constraints of having paper-based systems and in Uganda, it is difficult to coordinate the NES because of technical constraints (CLEAR-AA 2019b).

The public policy environment, in most instances, does not do enough to enable M\&E because in many African countries, their frameworks and systems were historically determined by a colonialism that was centred around domination and the facilitation of extraction. In post-independence Africa, this was exacerbated by a reliance on aid and structural adjustment programming, which anchored M\&E in the grip of accountability and compliance. As a result, accountability 
and learning have not been grounded and indigenised in the post-colonial context. Whilst this is changing, the process is a slow and complex one beset by the prevailing structural conditions that hinder change at scale.

Our analysis of African M\&E policies makes it clear that we need to be doing more to support their development and ensuring that they are context appropriate and do not simply mimic policies developed elsewhere by others. We have also concluded that data governance is an important, underaddressed priority and supporting countries to develop strategies and policies in this regard will become an important area of work for us.

\section{Leadership in the field of monitoring and evaluation}

Leadership plays a critical role in any institution or system, particularly if the intention of its interventions is transformational. Both M\&E are practices with enormous potential to drive improvements, transform systems and make them more effective, responsive, inclusive and participatory; however, if done badly, there is a serious risk that they will deflect attention, divert resources and undermine authentic accountability. For M\&E to realise their potential, they require inspired leadership at different levels. Such leadership should drive both monitoring, as a managerial function, and evaluation, as a strategic function, highlighting the value that evidence-based decision-making offers in the context of competing and conflicting priorities.

Unfortunately, with M\&E often seen as an administrative function at the service of political agendas, conflict at the political-administrative interface often scuppers even genuine attempts by state officials to drive a programme of authentic reflection and adaptation. Political leadership and politicaladministrative coherence are needed in championing the development of sustainable and effective M\&E systems. Embedding government-wide M\&E needs strong political will, dedicated staff and active participation across all levels and institutions of government (Twende Mbele 2018).

Providing leadership in M\&E can be thought of as having three dimensions. The first dimension is providing stewardship in integrating M\&E into existing processes and ensuring that they support and complement procedures in ways that add real value and enhance outcomes. The second dimension of leadership needed for effective M\&E is the ability to persuade internal and external stakeholders of the value and importance of evidence and deliberation in decision-making processes. This requires an ability to advocate for and promote participation of others and a commitment to improve results rather than the pursuit of private or sectional interests. The third dimension of leadership in M\&E is the ability to demonstrate effective practices and to show (rather than tell) how these improve results and enhance accountability. Demonstrating the value of $M \& E$ in practice moves the agenda out of the theoretical and academic realm into a more practical and meaningful mode where the value added becomes a motivator and incentive.

Since management practices are usually determined in the centres of government - in President's and Prime Minister's offices, development planning ministries and ministries of finance - having people work as integrators, persuaders and demonstrators in these institutions has proved particularly important.

We recognise that there is nothing coincidental about the spread of M\&E: it has been achieved intentionally and purposively and we draw from this realisation an appreciation of how important it is to forge in-country partnerships with champions and advocates who provide leadership now and in the future. The challenge is to develop a clearer, more actionable sense of exactly what kinds of support will make it easier for leaders to act to build M\&E systems and to put in place interventions that meet these needs.

\section{Culture: Curiosity, reflection and enquiry}

For $M \& E$ to add real value, they need an organisational culture that supports curiosity and enquiry. This is often also called an evaluative culture. An organisation with a strong evaluative culture is one that engages in self-reflection and self-examination and deliberately seeks evidence on what it is achieving. An organisation with a strong evaluative culture uses information on results to challenge itself and support what it is doing, depending on what is being discovered, and values candour, challenge and dialogue, not just superficially but in an authentic way. Government institutions require ongoing strengthening of evaluation culture as this will improve evaluation management skills (Twende Mbele 2019). Such organisations are engaged in evidence-based learning, allocating time and resources to learn in a structured, systematic way and encouraging the sharing of knowledge. Encouraging experimentation and a preparedness to take risks by seeking out new and different ways of doing business are also part of evaluative cultures (Mayne 2008).

Promoting an evaluative culture requires that senior management shows leadership and commitment by making regular, informed demand for results information and ensuring that capacity for results measurements and management is built. There also need to be strong organisational support structures with effective incentives, systems, practices and procedures and an outcome orientation across the organisational system (Mayne 2008). Perhaps more than anything, there need to be a tolerance for mistakes and a capacity to take on board lessons from failure, because without this, everything that happens in programmes gets framed as a success and learning is short circuited. For instance, the M\&E culture in Kenya according to Twende Mbele (2019:22) has a blend of doing things together (collaborative or clan culture), doing new things (adhocratic or creating culture), doing things fast (competition culture) and doing things right (control or hierarchy culture). 
In evaluation literature, the focus is increasingly on evaluative thinking, which is (Buckley et al. 2018):

[C]ritical thinking applied in the context of evaluation, motivated by an attitude of inquisitiveness and a belief in the value of evidence that involves identifying assumptions, posing thoughtful questions, pursuing deeper understanding through reflection and perspective making and informing decisions in preparation for action. (n.p.)

Both M\&E processes require a strong base in evaluative thinking to ensure that there is an appetite for what is learnt.

The general growing global consensus on the importance of M\&E brings with it an inherent danger: that the effort required to undertake these challenging and resourceintensive processes becomes over-regulated, ritualised and loses their meaning. They become a requirement that needs to be complied with rather than an opportunity for real-life learning and practical accountability. In some contexts, this takes on an ominous edge, morphing into what gets called 'malicious compliance'. In the South African context, the dangers long identified by Winston (1999), such as data gaming and goal displacement, remain common, despite an incremental shift in the direction of evaluation as learning in some places.

We appreciate that M\&E are profoundly political processes that echo and reflect the contexts in which they are undertaken. Amisi (2015) highlights this aspect, with the National Evaluation Plan (NEP) in South Africa widespread in terms of budget, number of people they reach and political significance. Politically, evidence produced by evaluation must be readily available to decision-makers in order for them to make an informed decision for continuity, modification or elimination of programmes (Lazaro 2015:15). Formal African governance systems were mostly shaped in the context of colonial control and domination and can be understood to be grafted on top of older organic structures and traditions. They are in many instances constrained and limited by this history which is complex and contradictory at every level, with a widespread tendency to Weberian forms of control and even bureaucratic authoritarianism 'command and control' are valued over 'enquire and reflect'. This is true of government systems as well as those of donors, which in many of the countries where CLEAR-AA operates have an outsized voice in how processes operate and what kind of evidence is solicited, how it is received and what kinds of organisational cultures are nurtured.

In contexts where authorities do not have a tradition of acknowledging difficulties or are committed to a particular - often triumphalist - narrative around performance, the culture needed for effective M\&E is unlikely to thrive. On the other hand, in situations characterised by robust political competition, which is increasingly the case in Africa, political parties are under pressure to prove their legitimacy by reporting positive results to the electorate, which puts another kind of pressure on M\&E systems to produce good news. Because public officeholders often have limited time to demonstrate the success of their leadership and garner political support from the electorate, there is a rush to provide evidence of performance and a distinct short-termism often comes to characterise public systems.

In neo-patrimonial states where there is extensive collusion amongst and between elements of the private sector and corrupt state officials to use public funds for private benefit, a climate of curiosity and enquiry is antithetical. Indeed, there is an inbuilt inclination to reject authentic enquiry into performance and expenditure. Monitoring and evaluation systems may, therefore, either not be prioritised or be designed in such a way that they could deflect attention from real problems (e.g. by encouraging a focus on 'safe' areas of enquiry), where they pose no real threat to corrupt practices.

For CLEAR-AA, we recognise that a culture of curiosity and enquiry is essential if the evidence is to be used in improving decision-making, and that doing so is neither simple nor quick. It is a complex, iterative process that takes many years, but that must be attempted as part of a broader reform and innovation agenda. For us, this means working in close partnership with country governments and civil societies and acting as a facilitator and adviser on a range of diverse projects that have a cumulative effect bigger than the sum of their parts. Central elements for us include, as noted above, policy development, so that there is an overarching framework to guide M\&E system strengthening efforts; research, so that system strengthening actions are based on an understanding of what currently exists; and capacitybuilding processes that take account of what capacity already exists and is thoughtful and careful to complement what else is being done.

\section{Conclusion: Partnerships for system strengthening}

There are five key lessons we have distilled from our experience in adopting a system approach to strengthen M\&E systems in our work in Anglophone African countries over the last decade. The first and most important of these is that the journey cannot be taken alone, and the domination of one or few partners or approaches working in enclaves will not achieve the result of a system-wide change. Adopting a collaborative, partnership-based approach to strengthen systems is therefore essential, and we need to draw on the strengths of various partners in development broadly, and in the $M \& E$ and evidence sectors more specifically, in order to address the challenges we have outlined in this article at various scales and levels of the system. Secondly, M\&E is not a stand-alone process and has no intrinsic value if separated from systems of decision-making. These are inextricably linked to budget and planning processes, and therefore efforts must continue to be directed towards integrating M\&E into planning and budgeting processes (whether in state- or non-state-led interventions). The third lesson is that strong leadership and the championing of the M\&E cause are catalytictostrengthen the performanceandinstitutionalisation 
of M\&E systems at country level. We, therefore, need to foster networks and communities of practice so that we can build a cadre of leaders who can act as champions, who are bolstered by a growing professionalism and deeper learning in the sector. This includes supporting the growth of voluntary organisations for professional evaluation (VOPES), 29 of which are registered with African Evaluation Association (AfrEA) across the continent. Other networks, such as Twende Mbele, represent a very unique network of countries cooperating to learn from each other, and others, such as the Africa Centre for Evidence and African Parliamentarians' Network on Development Evaluation (APNODE), are driving evidence use by policymakers and decision-makers.

Fourthly, capacity building must be considered as a comprehensive, whole-of-system intervention, and not to be confined to training only (and especially not only of the 'supply side'). Training and other kinds of capacity-building processes also need to be customised and tailored to the context, as systems are not all alike, and different incentives and disincentives that are part of the enabling environment need to be considered.

This links to the fifth takeaway, which is the need to support country governments in the development of indigenous policy frameworks that are crafted according to their particular governance and policy contexts. In recognition of the sui generis nature of governance systems, and the peculiarities of governance systems based on Africa's colonial past and the historical legacies they had left behind, we need to recognise the hybridity of post-colonial governance systems and work more deliberately to empower and invigorate an appreciation of this context, and be an enabler and advocate for the indigenisation of localised responses to the challenges they face in terms of evidence production and use. We must be facilitators of a transformational approach to evaluation, where an Africanised, contextually relevant approach to $M \& E$ is honoured, welcomed, valued and used.

In addition to experiencing the lessons, we have acknowledged the importance of reflection and learning from our work, and therefore we have prioritised research and learning (R\&L) as a distinctive area of focus in CLEARAA. This article is one example of the work that we continue to do in building a body of knowledge around Africa-centred approaches to establish NESs, which we hope will contribute to the strengthening of M\&E across the continent. In conclusion, what we are learning in our collaborative work with country partners and other organisations has allowed room for growth, in that we recognise the need for much more sustained research - both theoretical and empirical - on these areas of work, so that we get better what we are doing. Much needs to be done in documenting and building a solid body of knowledge, including case studies and theoretical frameworks, upon which we can improve our strategies and interventions at a system level.

\section{Acknowledgements Competing interests}

The authors declare that they have no financial or personal relationships that may have inappropriately influenced them in writing this article.

\section{Authors' contributions}

D.I.F. and C.M. contributed equally to the writing of this research article.

\section{Ethical considerations}

This article followed all ethical standards for research without direct contact with human or animal subjects.

\section{Funding information}

This research received no specific grant from any funding agency in the public, commercial or not-for-profit sectors.

\section{Data availability}

Data sharing is not applicable to this article as no new data were created or analysed in this study.

\section{Disclaimer}

The views and opinions expressed in this article are those of the authors and do not necessarily reflect the official policy or position of any affiliated agency of the authors.

\section{References}

Abrahams, M.A., 2015, 'A review of the growth of monitoring and evaluation in South Africa: Monitoring and evaluation as a profession, an industry and a governance tool', African Evaluation Journal 3(1), a142. https://doi.org/10.4102/aej.v3i1.142

Amisi, M.M., 2015, 'Improving the use of evaluative evidence through effective communication: Lessons from implementing the South African evaluation system', African Evaluation Journal 3(1), 7. https://doi.org/10.4102/aej.v3i1.109

Arnold, R.D. \& Wade, J.P., 2015, A definition of systems thinking: A systems approach, Steven's Institute, Hoboken, NJ.

Blaser Mapitsa, C. \& Khumalo, L., 2018, 'Diagnosing monitoring and evaluation capacity in Africa', African Evaluation Journal 6(1), a255. https://doi.org/10.4102/ aej.v6i1.255

Buckley, J., Archibald, T., Hargraves, M. \& Trochim, W.M., 2018, 'Defining and teaching evaluative thinking: Insights from research on critical thinking. American Journal of Evaluation 36(3), 375-388. https://doi.org/10.1177\%2F1098214015581706

CLEAR-AA, 2013, Demand and supply: Monitoring, evaluation, and performance management information and services in Anglophone sub-Saharan Africa: A synthesis of nine studies, viewed 12 May 2020, from https://www.theclearinitiative.org/sites/ clearinitiative/files/2016-04/Demand_and_Supply_Anglophone_Africa_2013.pdf.

CLEAR-AA, 2019a, Compass: Tracking monitoring and evaluation developments in Anglophone Africa, University of the Witwatersrand, Johannesburg, viewed 12 May 2020, from http://wiredspace.wits.ac.za/bitstream/handle/10539/28488/ CLEAR-AA\%20Compass. pdf?sequence=1\&isAllowed $=\mathrm{y}$.

CLEAR-AA, 2019b, Monitoring and evaluation in five African countries, University of the Witwatersrand, Johannesburg, viewed 12 May 2020, from http://wiredspace. wits.ac.za/bitstream/handle/10539/28232/CLEAR-AA_Diagnostics_Report_web. pdf?sequence $=1$ \&isAllowed $=y$.

Department of Planning, Monitoring and Evaluation, 2017, 'Evaluation of the National Evaluation System - Summary report', Department of Planning, Monitoring and Evaluation, Pretoria.

Fledderus, J., Brandsen, T. \& Honing, M., 2014, 'Restoring trust through the coproduction of public services: A theoretical elaboration', Public Management Review 16(3), 424-443. https://doi.org/10.1080/14719037.2013.848920

Goldman, I., Byamugisha, A., Gounou, A., Smith, L.R., Ntakumba, S., Sossou, D. et al., 2018 'The emergence of government evaluation systems in Africa: The case of Benin, Uganda and South Africa', African Evaluation Journal 6(1), 1-11, viewed from https://aejonline.org/index.php/aej/article/view/253/419. 
Lazaro, B., 2015, Comparative study on the institutionalisation of evaluation in Europe and Latin America, EUROsociAL, Brussels.

Masuku, N.W.K., 2015, 'A global overview of monitoring and evaluation (M\&E) and its meaning in the local government context of South Africa', Africa's Public Service Delivery \& Performance Review 3(2), a79. https://doi.org/10.4102/ apsdpr.v3i2.79

Mayne, J., 2008, 'Building an evaluative culture for effective evaluation and results management', in ILAC Brief No. 20, Institutional Learning and Change (ILAC) Initiative, Rome.

Merkle, C., 2016, 'UN women's experience with strengthening evaluation systems in Africa: Enhancing quantity, quality and use of evaluation', African Evaluation Journal 4(1), a127. https://doi.org/10.4102/aej.v4i1.127

Pazvakavambwa, A. \& Steyn, G.M., 2014, 'Implementing results-based management in the public sector of developing countries: What should be considered?', Mediterranean Journal of Social Sciences 5(20), 245-257 https://pdfs semanticscholar.org/6060/666b4adae54349da3703a58db76a169c83d3.pdf.

Phillips, S., 2018, Twende Mbele: Diagnostic on the demand and supply of evaluators in South Africa, Diagnostic Report, viewed 04 May 2020, from https://www.dpme. gov.za/keyfocusareas/gwmeSite/GovermentWide $\% 20 \mathrm{M} \% 20$ and $\% 20 \mathrm{E} /$ Diagnostic $\% 2$ report $\% 20$ Twende $\% 20$ Mbele $\% 20$ demand $\% 20$ and $\% 20$ supply $\% 20$ of $\% 20$ evaluation $\% 2021 \% 20$ January.pdf

Podems, D., Goldman, I. \& Jacob, C., 2014, 'Evaluator competencies: The South African government experience', The Canadian Journal of Program Evaluation 28(3), 71-85.
Tsai, M., 1999, State power, state embeddedness, and national development in less developed countries: A cross-national analysis, Studies in Comparative International Development 33(4), 66-88. https://doi.org/10.1007/BF02687524

Twende Mbele, 2018, Using M\&E to improve government performance and accountability, viewed 12 May 2020, from http://www.twendembele.org/wpcontent/uploads/2018/04/Twendle_Mbele_Using_ME_to_improve_ government_performance_and_accountability_2018.pdf.

Twende Mbele, 2019a, Diagnostic on the supply and demand of evaluators in Uganda, Benin and South Africa, viewed 12 May 2020, from http://www.twendembele. org/wp-content/uploads/2019/04/TWENDE-DS.pdf.

Twende Mbele, 2019b, Base study on the performance monitoring and evaluation culture in the public sector in Kenya, viewed 12 May 2020, from http://www. twendembele.org/wp-content/uploads/2020/01/ME-Culture-Kenya.pdf.

Umlaw, F. \& Chitepo, N., 2015, 'State and use of monitoring and evaluation systems in national and provincial departments', African Evaluation Journal 3(1), a134. https://doi.org/10.4102/ aej.v3i1.134

United Nations, c., 2020, High-level political forum on sustainable development sustainable development goals knowledge platform, viewed 04 May 2020, viewed from https://sustainabledevelopment.un.org/hlpf.

Winston, J., 1999, 'Performance indicators - Promises unmet: A response to Perrin', American Journal of Evaluation 20(1), 95-99. https://doi.org/10.1016/S10982140(99)80111-8 